\title{
The Riemann Problem \\ for a Two-Dimensional Hyperbolic System of Nonlinear Conservation Laws: Multiplication of Distribution Solutions
}

\author{
Jiaxin $\mathrm{Hu}$
}

\begin{abstract}
In the papers [6 - 8], the author has constructed the Riemann solutions to a twodimensional hyperbolic system of nonlinear conservation laws for any piecewise constant initial data having two discontinuity rays with origin as vertex. It has been found that, for some initial data, the Riemann solutions no longer lie in $L_{\text {loc }}^{\infty}\left(\mathbb{R}^{2} \times \mathbb{R}_{+}\right)$, and the non-classical waves (labelled as Dirac-contact waves) have arisen. But it remains open in [6 - 8] to verify that the non-classical solutions constructed satisfy the system considered. In the present paper, we borrow the new mathematical theory of generalized functions, chiefly initiated by $\mathrm{J}$. $F$. Colombeau and Rosinger, to deal with the diffculty of the multiplication of distribution solutions. The non-classical Riemann solutions we constructed in [6-8] satisfy the system in the sense of association. The present paper provides a good example of applications for this new mathematical theory in powerfully handling the product of generalized functions.
\end{abstract}

Keywords: Two-dimensional conservation laws, Riemann problems, non-classical waves, multiplication of distributions

AMS subject classiflcation: 35 L 65

\section{Introduction}

We are concerned with the two-dimensional hyperbolic system of nonlinear conservation laws

$$
\left.\begin{array}{l}
u_{t}+(u v)_{y}=0 \\
v_{t}+(u v)_{x}=0
\end{array}\right\} \quad\left((x, y, t) \in \mathbb{R}^{2} \times \mathbb{R}_{+}\right)
$$

with initial data

$$
\left.(u, v)\right|_{\imath=0}= \begin{cases}\left(u_{2}, v_{2}\right) & \text { for } x, y>0 \\ \left(u_{1}, v_{1}\right) & \text { otherwise }\end{cases}
$$

where $\left(u_{i}, v_{i}\right)(i=1,2)$ are constant states. We call (1.1), (1.2) a Riemann problem.

System (1.1) is the special form of the mathematical simplification of the twodimensional linearized model of the cochlea. We recall that in the absence of fluid Jiaxin Hu: Academia Sinica, Wuhan Inst. Phys. Math., Wuhan 430071, P.O. Box 71010, P.R. China; e-mail: jxhu@math.whcnc.ac.cn 
viscosity the linearized equations of motion in the fluid-filled inner ear (cochlea) read

$$
\begin{aligned}
& \rho \frac{\partial u}{\partial t}+\frac{\partial p}{\partial y}=0 \\
& \rho \frac{\partial v}{\partial t}+\frac{\partial p}{\partial x}=0 \\
& \frac{\partial u}{\partial y}+\frac{\partial v}{\partial x}=0
\end{aligned}
$$

where $(u, v)$ and $p$ stand for the fluid velocity and pressure, respectively, while $\rho$ is the (constant) density (see [9] and the references therein). Neglecting (1.5) and assuming $p=\rho u v$, one finds that $(1.3),(1.4)$ are reduced to the system (1.1).

In the papers [6 - 8] we constructed the Riemann solutions to (1.1) for any piecewise constant initial data (1.2). In particular, some Riemann solutions contain the nonclassical waves (labelled as the Dirac-contact waves). In other words, these Riemann solutions no longer lie in $L_{\text {loc }}^{\infty}\left(\mathbb{R}^{2} \times \mathbb{R}_{+}\right)$, the space of locally bounded functions on $\mathbb{R}^{2} \times \mathbb{R}_{+}$, although the initial data belong to $L^{\infty}\left(\mathbb{R}^{2}\right)$. This is distinctly different from that people investigated before (see [14]). In [6 - 8], the non-classical Riemann solutions to (1.1), (1.2) were shown to possess high singularity on the Dirac-contact waves and can be viewed as the bounded functions in $L^{\infty}\left(\mathbb{R}^{2} \times \mathbb{R}_{+}\right)$plus the Schwartz generalized functions supported on the Dirac-contact waves. At this time, there arises a concurrent problem of how to define the product of two Schwartz generalized functions. According to L. Schwartz's theory, it is impossible to define the product of two arbitrary Schwartz generalized functions since the space of all Schwartz generalized functions is not an algebraic one [13]. Because of this difficulty, we failed to verify in $[6-8]$ that the non-classical Riemann solutions constructed satisfy the system (1.1). In the present paper, we apply a new mathematical theory of generalized functions, chiefly introduced by J. F. Colombeau and Rosinger $[1,2,12]$, to dealing with the multiplication of the distributions appearing in the non-classsical Riemann solutions to (1.1), (1.2), and have fully solved the open problem left in $[6-8]$. This new theory of generalized functions is the extension of Schwartz generalized function theory and allows us to define the product of two arbitrary Schwartz generalized functions. It was later developed by Oberguggenberger $[10,11]$ and other people (see, e.c., $[2-4,5,15]$ ). We remark in passing here that in recent years, the non-classical waves have attracted great interest, and many people have paid attention to investigating them (see [16 - 21]).

The program of this paper is as follows:

In Section 2, for the reader's convenience, we shall give a glimpse of this new mathematical theory of generalized functions and then interpret in what sense the Riemann solutions we constructed in [6 - 8] satisfy (1.1), (1.2) in the framework of this new mathematical theory (cf. (2.3), (2.4)). In Section 3 we only pay our attention to a representative case that $u_{1} \geq 0 \geq u_{2}$ and $v_{1} \geq 0 \geq v_{2} \quad\left(u_{1} \neq u_{2}, v_{1} \neq v_{2}\right.$ and $\left.u_{1} v_{1} \neq u_{2} v_{2}\right)$ (at this time, both $u$ and $v$ are singular on the Dirac-contact waves and there arises the difficulty of the product of distributions). Then we verify that the non-classical Riemann solutions satisfy (1.1) in the sense of association. 


\section{The new generalized function space $\mathcal{G}(\Omega)$}

In this section we briefly describe the definition of the new generalized function space $\mathcal{G}(\Omega)$ introduced by Colombeau and Rosinger $[1,2,12]$.

Let $\Omega$ be an open set in $\mathbb{R}^{n}$ and we denote by $\mathcal{E}_{M}[\Omega]$ the set of all the maps $R(\varepsilon, x)$ : $(0,1] \times \Omega \rightarrow \mathcal{C}$ such that:

(i) For any $\varepsilon>0$, the map $R(\varepsilon, x)$ is a $C^{\infty}$-function of the variable $x \in \Omega$.

(ii) If $D=\frac{\partial^{|k|}}{\partial x_{1}^{k_{1}} \ldots \partial x_{n}^{k_{n}}}$ is any partial derivation operator and if $K$ is any compact subset of $\Omega$, then there exists an integer $N$ and two constants $C>0$ and $\eta>0$ with $0<\eta<1$ such that $\sup _{x \in K}|D R(\varepsilon, x)| \leq \frac{C}{\varepsilon^{N}}$ if $0<\varepsilon<\eta$.

Next let $\mathcal{N}[\Omega]$ be the set of all elements $R \in \mathcal{E}_{M}[\Omega]$ with the property that, for all $D$ and $K$ as above, we have an integer $N$ such that for all $q \geq N$ there exist $C_{q}, \eta_{q}>0$ with $\sup _{x \in K}|D R(\varepsilon, x)| \leq C_{q} \varepsilon^{q}$ if $0<\varepsilon<\eta_{q}$. Obviously, $\mathcal{N}[\Omega]$ is an ideal of the algebra $\mathcal{E}_{M}[\Omega]$. The new generalized function space $\mathcal{G}(\Omega)$ is defined as the quotient algebra

$$
\mathcal{G}(\Omega)=\mathcal{E}_{M}[\Omega] / \mathcal{N}[\Omega]
$$

The operations in $\mathcal{G}(\Omega)$ such as differentiation, addition and multiplication are those naturally defined on representatives; in particular, multiplication is possible in $\mathcal{G}(\Omega)$ because $\mathcal{N}[\Omega]$ is an ideal of the algebra $\mathcal{E}_{M}[\Omega]$.

Two generalized functions $G_{1}, G_{2} \in \mathcal{G}(\Omega)$ are said to be associated (in notation: $G_{1} \approx G_{2}$ ) if there exist some representatives $R_{1}$ and $R_{2}$ of $G_{1}$ and $G_{2}$, respectively, such that for all $\psi \in \mathcal{D}(\Omega)$

$$
\lim _{\varepsilon \rightarrow 0} \int_{\Omega}\left(R_{1}(\varepsilon, x)-R_{2}(\varepsilon, x)\right) \psi(x) d x=0
$$

An element $G \in \mathcal{G}(\Omega)$ is said to have a distribution $T \in \mathcal{D}^{\prime}(\Omega)$ as macroscopic aspect, if $G \approx T$, i.e. for all $\psi \in \mathcal{D}(\Omega)$

$$
\lim _{\varepsilon \rightarrow 0} \int_{\Omega} R(\varepsilon, x) \psi(x) d x=\langle T(\lambda), \psi(\lambda-x)\rangle
$$

for some representative $R$ of $G$.

Now we are in a position to give in what sense the Riemann solutions we constructed in $[6-8]$ satisfy (1.1), (1.2). First of all, we note that (1.1), (1.2) are invariant under the self-similar transformation

$$
\left.\begin{array}{l}
x \rightarrow \alpha x^{\prime} \\
y \rightarrow \alpha y^{\prime} \\
t \rightarrow \alpha t^{\prime}
\end{array}\right\} \quad(\alpha>0)
$$

We should seek self-similar solutions of the form

$$
(u(x, y, t), v(x, y, t))=(u(\xi, \eta), v(\xi, \eta)) \quad\left(\xi=\frac{x}{t}, \eta=\frac{y}{t}\right) .
$$


Thus (1.1) changes into

$$
\left.\begin{array}{rl}
-\xi u_{\xi}-\eta u_{\eta}+(u v)_{\eta} & =0 \\
-\xi v_{\xi}-\eta v_{\eta}+(u v)_{\xi} & =0
\end{array}\right\} \quad\left((\xi, \eta) \in \mathbb{R}^{2}\right)
$$

and (1.2) into

$$
(u(\xi, \eta), v(\xi, \eta)) \rightarrow \begin{cases}\left(u_{2}, v_{2}\right) & \text { for } \xi \rightarrow \infty, \eta \rightarrow \infty \\ \left(u_{1}, v_{1}\right) & \text { for } \xi \rightarrow \infty, \eta \rightarrow-\infty \text { or } \xi \rightarrow-\infty,|\eta| \rightarrow \infty\end{cases}
$$
if

Definition 2.1. The Riemann solution $(U, V) \in \mathcal{G}\left(\mathbb{R}^{2}\right)$ is said to satisfy $(1.1),(1.2)$

$$
\left.\begin{array}{r}
-\xi U_{\xi}-\eta U_{\eta}+(U V)_{\eta} \approx 0 \\
-\xi V_{\xi}-\eta V_{\eta}+(U V)_{\xi} \approx 0
\end{array}\right\}
$$

The sense in which $(U, V) \in \mathcal{G}(\mathbb{R})$ satisfies the initial data (1.2) should be investigated carefully. In the present paper, we simply give the following justification:

Let $\left(R_{u}(\varepsilon, \xi, \eta), R_{v}(\varepsilon, \xi, \eta)\right)$ be any representative of $(U, V)$. If

$$
\left(R_{u}(\varepsilon, \xi, \eta), R_{v}(\varepsilon, \xi, \eta)\right)
$$

converges to some function pair

$$
(u(\xi, \eta), v(\xi, \eta))=\left(u\left(\frac{x}{t}\right), v\left(\frac{y}{t}\right)\right) \in L_{l o c}^{\infty}\left(\mathbb{R}^{2} \times \mathbb{R}_{+}\right)
$$

in $\left(\mathcal{D}_{1}\left(\mathbb{R}^{2}\right)\right)^{\prime}$ as $\varepsilon \rightarrow 0$, i.e.

$$
\begin{aligned}
& \lim _{\varepsilon \rightarrow 0} \iint_{\mathbb{R}^{2}} R_{u}(\varepsilon, \xi, \eta) \varphi(\xi, \eta) d \xi d \eta=\iint_{\mathbb{R}^{2}} u(\xi, \eta) \varphi(\xi, \eta) d \xi d \eta \\
& \lim _{\varepsilon \rightarrow 0} \iint_{\mathbb{R}^{2}} R_{v}(\varepsilon, \xi, \eta) \varphi(\xi, \eta) d \xi d \eta=\iint_{\mathbb{R}^{2}} v(\xi, \eta) \varphi(\xi, \eta) d \xi d \eta
\end{aligned}
$$

for each $\varphi \in \mathcal{D}_{1}\left(\mathbb{R}^{2}\right)$, where

$$
\mathcal{D}_{1}\left(\mathbb{R}^{2}\right)=\left\{\varphi \in C_{0}^{\infty}\left(\mathbb{R}^{2}\right): \varphi(\xi, 0)=0 \text { for all } \xi \in \mathbb{R} \text { and } \varphi(0, \eta)=0 \text { for all } \eta \in \mathbb{R}\right\}
$$

and if

$$
u\left(\frac{x}{t}, \frac{y}{t}\right) \rightarrow u_{0}(x, y) \quad \text { and } \quad v\left(\frac{x}{t}, \frac{y}{t}\right) \rightarrow v_{0}(x, y) \quad \text { in } L_{l o c}^{1}\left(\mathbb{R}^{2}\right)
$$

as $t \rightarrow 0+$, we say that $(U, V) \in \mathcal{G}\left(\mathbb{R}^{2}\right)$ satisfies (1.2).

We note that $(2.4)_{1}$ is feasible for the Riemann solution to the system (1.1) since the non-classical wave appearing in the Riemann solution develops only from the discontinuity line $x=0, y \geq 0$ or $y=0, x \geq 0$ of the initial data as time evolves. 


\section{Verification of (2.3), (2.4) for non-classical Riemann solutions constructed in [6-8]}

In this section we shall verify that the non-classical Riemann solutions we constructed in [6 - 8] satisfy (2.3), (2.4). We only pay our attention to the case that $u_{1} \geq 0 \geq u_{2}$ and $v_{1} \geq 0 \geq v_{2} \quad\left(u_{1} \neq u_{2}, v_{1} \neq v_{2}\right.$ and $\left.u_{1} v_{1} \neq u_{2} v_{2}\right)$. Other cases can be treated similarly.

At this time, the Riemann solution to (1.1), (1.2) is constructed as follows [6 - 8]:

(i) By the initial condition (2.2), there exists a sufficiently large circle in the $(\xi, \eta)$ plane, outside which the solution to (1.1), (1.2) looks like that of the Riemann problem for corresponding one-dimensional system of conservation laws, and what we should do is to determine the interaction in the circle of waves coming from infinity.

(ii) There exists a Dirac-contact wave (denoted by $\delta_{1}$ ), which comes from infinity and is given by $\xi=0, \eta>0$, since $u_{1} \geq 0 \geq u_{2}\left(u_{1}>u_{2}\right)$ (see [6 - 8]). Also, there is another Dirac-contact wave (denoted by $\delta_{2}$ ), which comes from infinity and is given by $\eta=0, \xi>0$, since $v_{1} \geq 0 \geq v_{2}\left(v_{1}>v_{2}\right)$.

(iii) $\delta_{1}$ and $\delta_{2}$ hit at the point $(0,0)$ (see the following figure).

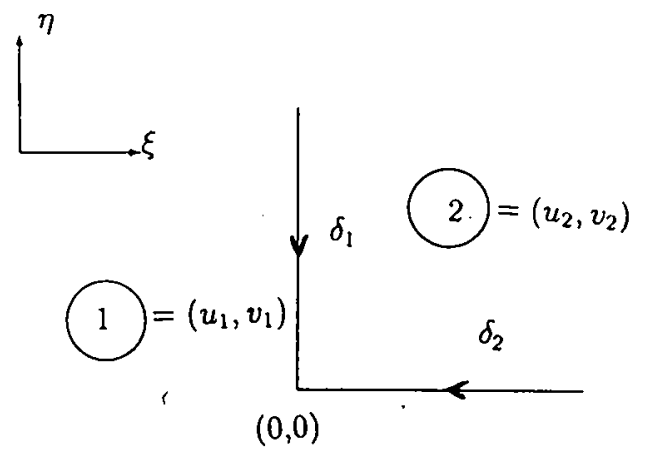

Next we give the expressions of the elements $R_{u}$ and $R_{v}$ of the Riemann solution $(U, V) \in \mathcal{G}\left(\mathbb{R}^{2}\right)$ and verify that for $\psi \in C_{0}^{\infty}\left(\mathbb{R}^{2}\right)$

$$
\begin{aligned}
& \lim _{\varepsilon \rightarrow 0} \iint_{\mathbb{R}^{2}}\left[R_{u}(\varepsilon, \xi, \eta)\left((\xi \psi)_{\xi}+(\eta \psi)_{\eta}\right)-R_{u}(\varepsilon, \xi, \eta) R_{v}(\varepsilon, \xi, \eta) \psi_{\eta}\right] d \xi d \eta=0 \\
& \lim _{\varepsilon \rightarrow 0} \iint_{\mathbb{R}^{2}}\left[R_{v}(\varepsilon, \xi, \eta)\left((\xi \psi)_{\xi}+(\eta \psi)_{\eta}\right)-R_{u}(\varepsilon, \xi, \eta) R_{v}(\varepsilon, \xi, \eta) \psi_{\xi}\right] d \xi d \eta=0 .
\end{aligned}
$$

We first consider $u_{1} v_{1}=0$ and then discuss the case that $u_{1} v_{1} \neq 0$.

3.1 The case $u_{1} v_{1}=0$. Without loss of generality, we assume that $u_{1}=0$ and let

$$
\left.\begin{array}{l}
\bar{u}(\xi, \eta)=u_{2} H(\xi, \eta) \\
\bar{v}(\xi, \eta)=v_{1}+\left(v_{2}-v_{1}\right) H(\xi, \eta)
\end{array}\right\}
$$

where $H(\xi, \eta)=1$ for $\xi, \eta>0$ and $H(\xi, \eta)=0$ otherwise. From the construction above, we know that the Riemann solution to (1.1), (1.2) equals

$$
(\bar{u}(\xi, \eta), \bar{v}(\xi, \eta))= \begin{cases}\left(u_{2}, v_{2}\right) & \text { for } \xi>0, \eta>0 \\ \left(u_{1}, v_{1}\right)=\left(0, v_{1}\right) & \text { for } \xi<0,-\infty<\eta<\infty \text { or } \xi>0, \eta<0 .\end{cases}
$$


But $(\bar{u}(\xi, \eta), \bar{v}(\xi, \eta))$ is not a solution to (1.1), (1.2) in the sense of distributions (or association). In fact, $(2.1)$ never holds true for $(\bar{u}(\xi, \eta), \bar{v}(\xi, \eta))$ in the sense of distributions (or association) since

$$
\left.\begin{array}{l}
\iint_{\mathbb{R}^{2}}\left[\left((\xi \psi)_{\xi}+(\eta \psi)_{\eta}\right) \bar{u}(\xi, \eta)-\psi_{\eta} \bar{u}(\xi, \eta) \bar{v}(\xi, \eta)\right] d \xi d \eta=u_{2} v_{2} \int_{0}^{\infty} \psi(\xi, 0) d \xi \\
\iint_{\mathbb{R}^{2}}\left[\left((\xi \psi)_{\xi}+(\eta \psi)_{\eta}\right) \bar{v}(\xi, \eta)-\psi_{\xi} \bar{u}(\xi, \eta) \bar{v}(\xi, \eta)\right] d \xi d \eta=u_{2} v_{2} \int_{0}^{\infty} \psi(0, \eta) d \eta
\end{array}\right\}
$$

and $u_{2} v_{2} \neq u_{1} v_{1}=0$. Thus the Riemann solution to (1.1), (1.2) is much more than $(\bar{u}(\xi, \eta), \bar{v}(\xi, \eta))$. The right sides of (3.4) carry enough information for us to give the expressions of the elements $R_{u}$ and $R_{v}$ of the Riemann solution $(U, V) \in \mathcal{G}\left(\mathbb{R}^{2}\right)$ to (1.1), (1.2).

Let $T_{1}, T_{2}$ be two bounded linear functionals given by

$$
\begin{aligned}
& \left\langle T_{1}, \psi\right\rangle=\int_{0}^{\infty} r(\xi) \psi(\xi, 0) d \xi \\
& \left\langle T_{2}, \psi\right\rangle=\int_{0}^{\infty} s(\eta) \psi(0, \eta) d \eta
\end{aligned}
$$

for $\psi \in C_{0}^{\infty}\left(\mathbb{R}^{2}\right)$, where $r$ and $s$ are some continuous functions on $[0, \infty)$ yet to be determined below. It is easily seen that $T_{2}$ is supported on the open half $\eta$-axis $\xi=$ $0, \eta>0$ and $T_{1}$ is supported on the open half $\xi$-axis $\eta=0, \xi>0$. Now we define

$$
\begin{aligned}
& R_{u}(\varepsilon, \xi, \eta)=\left(\bar{u} * w_{1 \varepsilon}\right)(\xi, \eta)+u_{2} v_{2}\left(T_{1} * w_{1 \varepsilon}\right)(\xi, \eta) \\
& R_{v}(\varepsilon, \xi, \eta)=\left(\bar{v} * w_{2 \varepsilon}\right)(\xi, \eta)+u_{2} v_{2}\left(T_{2} * w_{2 \varepsilon}\right)(\xi, \eta)
\end{aligned}
$$

where $w_{i \varepsilon}(\xi, \eta)=\frac{1}{e^{2}} \theta_{i}\left(\frac{\xi}{\varepsilon}\right) \phi_{i}\left(\frac{\eta}{\varepsilon}\right)$ and $\theta_{i}, \phi_{i} \in \mathcal{D}(\mathbb{R})$ with $\int_{\mathbb{R}} \theta_{i}(\xi) d \xi=\int_{\mathbb{R}} \phi_{i}(\xi) d \xi=$ $1(i=1,2)$. We rewrite $(3.7)$ as

$$
\begin{aligned}
R_{u}(\varepsilon, \xi, \eta)= & u_{2} \int_{-\xi / \varepsilon}^{\infty} \theta_{1}(x) d x \int_{-\eta / \epsilon}^{\infty} \phi_{1}(y) d y \\
& +u_{2} v_{2} \frac{1}{\varepsilon} \phi_{1}\left(-\frac{\eta}{\varepsilon}\right) \int_{-\xi / \epsilon}^{\infty} r(\xi+\varepsilon x) \theta_{1}(x) d x \\
R_{v}(\varepsilon, \xi, \eta)= & v_{1}+\left(v_{2}-v_{1}\right) \int_{-\xi / \epsilon}^{\infty} \theta_{2}(x) d x \int_{-\eta / \epsilon}^{\infty} \phi_{2}(y) d y \\
& +u_{2} v_{2} \frac{1}{\varepsilon} \theta_{2}\left(-\frac{\xi}{\varepsilon}\right) \int_{-\eta / \epsilon}^{\infty} s(\eta+\varepsilon y) \phi_{2}(y) d y
\end{aligned}
$$


From (3.1), (3.2) we can determine $r$ and $s$. To do this, we compute that for $\psi \in$ $C_{0}^{\infty}\left(\mathbb{R}^{2}\right),(3.8)$ and $(3.9)$ give

$$
\begin{aligned}
& \lim _{\varepsilon \rightarrow 0} \iint_{\mathbb{R}^{2}} R_{u}(\varepsilon, \xi, \eta) \psi(\xi, \eta) d \xi d \eta \\
& =u_{2} \int_{0}^{\infty} \int_{0}^{\infty} \psi(\xi, \eta) d \xi d \eta+u_{2} v_{2} \int_{0}^{\infty} r(\xi) \psi(\xi, 0) d \xi \\
& \lim _{\varepsilon \rightarrow 0} \iint_{\mathbb{R}^{2}} R_{v}(\varepsilon, \xi, \eta) \psi(\xi, \eta) d \xi d \eta \\
& =v_{1} \iint_{\mathbb{R}^{2}} \psi(\xi, \eta) d \xi d \eta+\left(v_{2}-v_{1}\right) \int_{0}^{\infty} \int_{0}^{\infty} \psi(\xi, \eta) d \xi d \eta \\
& \quad+u_{2} v_{2} \int_{0}^{\infty} s(\eta) \psi(0, \eta) d \eta
\end{aligned}
$$

and

$$
\begin{aligned}
& \lim _{\varepsilon \rightarrow 0} \iint_{\mathbb{R}^{2}} R_{u}(\varepsilon, \xi, \eta) R_{v}(\varepsilon, \xi, \eta) \psi(\xi, \eta) d \xi d \eta \\
& =u_{2} v_{2} \int_{0}^{\infty} \int_{0}^{\infty} \psi(\xi, \eta) d \xi d \eta+u_{2}^{2} v_{2} \lim _{\varepsilon \rightarrow 0} \iint_{\mathbb{R}^{2}} \psi(\xi, \eta) \frac{1}{\varepsilon} \theta_{2}\left(-\frac{\xi}{\varepsilon}\right) \\
& \times \int_{-\xi / \varepsilon}^{\infty} \theta_{1}(x) d x \int_{-\eta / \varepsilon}^{\infty} \phi_{1}(y) d y \cdot \int_{-\eta / \varepsilon}^{\infty} s(\eta+\varepsilon y) \phi(y) d y d \xi d \eta \\
& +u_{2} v_{2} \lim _{\varepsilon \rightarrow 0} \iint_{\mathbb{R}^{2}} \psi(\xi, \eta) \frac{1}{\varepsilon} \phi_{1}\left(-\frac{\eta}{\varepsilon}\right) \int_{-\xi / \epsilon}^{\infty} r(\xi+\varepsilon x) \theta_{1}(x) d x \\
& \times\left(v_{1}+\left(v_{2}-v_{1}\right) \int_{-\xi / \varepsilon}^{\infty} \theta_{2}(x) d x \cdot \int_{-\eta / \epsilon}^{\infty} \phi_{2}(y) d y\right) d \xi d \eta \\
& +u_{2}^{2} v_{2}^{2} \lim _{\varepsilon \rightarrow 0} \iint_{\mathbb{R}^{2}} \psi(\xi, \eta) \frac{1}{\varepsilon} \phi_{1}\left(-\frac{\eta}{\varepsilon}\right) \frac{1}{\varepsilon} \theta_{2}\left(-\frac{\xi}{\varepsilon}\right) \int_{-\xi / \epsilon}^{\infty} r(\xi+\varepsilon x) \theta_{1}(x) d x \\
& \times \int_{-\eta / \epsilon}^{\infty} s(\eta+\varepsilon y) \phi_{2}(y) d y d \xi d \eta \\
& =u_{2} v_{2} \int_{0}^{\infty} \int_{0}^{\infty} \psi(\xi, \eta) d \xi d \eta+u_{2}^{2} v_{2} \cdot A \int_{0}^{\infty} s(\eta) \psi(0, \eta) d \eta
\end{aligned}
$$




$$
+u_{2} v_{2}\left(v_{1}+\left(v_{2}-v_{1}\right) B\right) \int_{0}^{\infty} r(\xi) \psi(\xi, 0) d \xi+u_{2}^{2} v_{2}^{2} A B r(0) s(0) \psi(0,0)
$$

where $A=\int_{R} \theta_{2}(\xi)\left(\int_{\xi}^{\infty} \theta_{1}(x) d x\right) d \xi$ and $B=\int_{R} \phi_{1}(\eta)\left(\int_{\eta}^{\infty} \phi_{2}(y) d y\right) d \eta$. Appropriately choosing $\theta_{i}$ and $\phi_{i} \quad(i=1,2)$ such that $A=0$ and $B=\frac{v_{1}}{v_{1}-v_{2}}$, one gets at once from (3.12) that

$$
\lim _{\varepsilon \rightarrow 0} \iint_{\mathbb{R}^{2}} R_{u}(\varepsilon, \xi, \eta) R_{v}(\varepsilon, \xi, \eta) \psi(\xi, \eta) d \xi d \eta=u_{2} v_{2} \int_{0}^{\infty} \int_{0}^{\infty} \psi(\xi, \eta) d \xi d \eta
$$

Using (3.10) and (3.13), it follows from (3.1) that $\int_{0}^{\infty}\left(r(\xi)-\xi r^{\prime}(\xi)+1\right) \psi(\xi, 0) d \xi=0$ which implies that $r(\xi)-\xi r^{\prime}(\xi)+1=0(\xi \geq 0)$, i.e., $r(\xi)=a \xi-1(\xi \geq 0)$. Here $a$ is any constant.

Similarly, from (3.11) and (3.13) we deduce from (3.2) that $s(\eta)=b \eta-1 \quad(\eta \geq 0)$, with $b$ arbitrary constant, for the $\theta_{i}$ and $\phi_{i}(i=1,2)$ chosen above. Therefore, $(U, \bar{V}) \in$ $\mathcal{G}\left(\mathbb{R}^{2}\right)$ satisfies (2.3). And (2.4) is trivial. Thus we have proved that $(U, V) \in \mathcal{G}\left(\mathbb{R}^{2}\right)$, with $\bar{u}(\xi, \eta)+u_{2} v_{2} T_{1}$ and $\bar{v}(\xi, \eta)+u_{2} v_{2} T_{2}$ as their macroscopic aspects, respectively, satisfies (1.1) and (1.2) in the sense of association when $u_{1}=0>u_{2}$ and $v_{1} \geq 0>v_{2}$.

Remarks 1. We have infinitely many different Schwartz generalized functions $T_{1}$ and $T_{2}$ defined above since $r(\xi)=a \xi-1$ and $s(\eta)=b \eta-1$, and so the macroscopic aspects of $U$ and $V$ are infinitely numerous. In this sense the Riemann solution to (1.1), (1.2) is not unique.

2. The elements of the Riemann solution $(U, V) \in \mathcal{G}\left(\mathbb{R}^{2}\right)$ strongly depend on the choices of regularization process, which eventually depend on the system and the initial datum. Thus the ambiguity is removed in defining the multiplication of two distributions $U, V \in \mathcal{G}\left(\mathbb{R}^{2}\right)$ (see (3.13)).

3.2 The case $u_{1} v_{1} \neq 0$. We set

$$
\begin{aligned}
& \bar{u}(\xi, \eta)=u_{1}+\left(u_{2}-u_{1}\right) H(\xi, \eta) \\
& \bar{v}(\xi, \eta)=v_{1}+\left(v_{2}-v_{1}\right) H(\xi, \eta)
\end{aligned}
$$

and

$$
\begin{aligned}
& \left\langle T_{1}, \psi\right\rangle=\int_{0}^{\infty}(a \xi-1) \psi(\xi, 0) d \xi \\
& \left\langle T_{2}, \psi\right\rangle=\int_{0}^{\infty}(b \eta-1) \psi(0, \eta) d \eta
\end{aligned}
$$

where $H$ is the same as (3.3) and $a, b$ are arbitrary constants. As above, we can define $\bar{U}, \bar{V} \in \mathcal{G}\left(\mathbb{R}^{2}\right)$ with $\bar{u}(\xi, \eta)+\rho T_{1}$ and $\bar{v}(\xi, \eta)+\rho T_{2}$ as their macroscopic aspects, $\rho=$ 
$u_{2} v_{2}-u_{1} v_{1} \neq 0$. Let $R_{u}$ and $R_{v}$ be representatives of $\bar{U}$ and $\bar{V}$, respectively, where

$$
\begin{aligned}
\bar{R}_{u}(\varepsilon, \xi, \eta)= & \left(\bar{u} * w_{1 \epsilon}\right)(\xi, \eta)+\rho\left(T_{1} * w_{1 \varepsilon}\right)(\xi, \eta) \\
= & u_{1}+\left(u_{2}-u_{1}\right) \int_{-\xi / \varepsilon}^{\infty} \theta_{1}(x) d x \int_{-\eta / \epsilon}^{\infty} \phi_{1}(y) d y \\
& +\rho \frac{1}{\varepsilon} \phi_{1}\left(-\frac{\eta}{\varepsilon}\right) \int_{-\xi / \varepsilon}^{\infty}(a \xi+a \varepsilon x-1) \theta_{1}(x) d x \\
\bar{R}_{v}(\varepsilon, \xi, \eta)= & \left(\bar{v} * w_{2 \varepsilon}\right)(\xi, \eta)+\rho\left(T_{2} * w_{2 \varepsilon}\right)(\xi, \eta) \\
= & v_{1}+\left(v_{2}-v_{1}\right) \int_{-\xi / \epsilon}^{\infty} \theta_{2}(x) d x \int_{-\eta / \epsilon}^{\infty} \phi_{2}(y) d y \\
& +\rho \frac{1}{\varepsilon} \theta_{2}\left(-\frac{\xi}{\varepsilon}\right) \int_{-\eta / \epsilon}^{\infty}(b \eta+\varepsilon b y-1) \phi_{2}(y) d y .
\end{aligned}
$$

However, $(\bar{U}, \bar{V}) \in \mathcal{G}\left(\mathbb{R}^{2}\right)$ does not satisfy (1.1) in the sense of association. As a matter of fact, from (3.16) we compute that for $\psi \in C_{0}^{\infty}\left(\mathbb{R}^{2}\right)$

$$
\begin{aligned}
\lim _{\varepsilon \rightarrow 0} \iint_{\mathbb{R}^{2}} \bar{R}_{u}(\varepsilon, \xi, \eta) \psi(\xi, \eta) d \xi d \eta \\
=\iint_{\mathbb{R}^{2}}\left(u_{1}+\left(u_{2}-u_{1}\right) H(\xi, \eta)\right) \psi(\xi, \eta) d \xi d \eta \\
\quad+\lim _{\varepsilon \rightarrow 0} \frac{\rho}{\varepsilon} \iint_{\mathbb{R}^{2}} \psi(\xi, \eta) \phi_{1}\left(-\frac{\eta}{\varepsilon}\right) \int_{-\xi / \varepsilon}^{\infty}(a \xi+a \varepsilon x-1) \theta_{1}(x) d x \\
=u_{1} \iint_{\mathbb{R}^{2}} \psi(\xi, \eta) d \xi d \eta \\
\quad+\left(u_{2}-u_{1}\right) \int_{0}^{\infty} \int_{0}^{\infty} \psi(\xi, \eta) d \xi d \eta+\rho \int_{0}^{\infty}(a \xi-1) \psi(\xi, 0) d \xi
\end{aligned}
$$

and

$$
\begin{aligned}
& \lim _{\varepsilon \rightarrow 0} \iint_{\mathbb{R}^{2}} \bar{R}_{u}(\varepsilon, \xi, \eta) \bar{R}_{v}(\varepsilon, \xi, \eta) \psi(\xi, \eta) d \xi d \eta \\
& =u_{1} v_{1} \iint_{\mathbb{R}^{2}} \psi(\xi, \eta) d \xi d \eta+\left(u_{2} v_{2}-u_{1} v_{1}\right) \int_{0}^{\infty} \int_{0}^{\infty} \psi(\xi, \eta) d \xi d \eta \\
& \quad+\rho\left(u_{1}+\left(u_{2}-u_{1}\right) A\right) \int_{0}^{\infty}(b \eta-1) \psi(0, \eta) d \eta
\end{aligned}
$$




$$
+\rho\left(v_{1}+\left(v_{2}-v_{1}\right) B\right) \int_{0}^{\infty}(a \xi-1) \psi(\xi, 0) d \xi+\rho^{2} A B \psi(0,0)
$$

where $A=\int_{\mathbb{R}} \theta_{2}(\xi)\left(\int_{\xi}^{\infty} \theta_{1}(x) d x\right) d \xi$ and $B=\int_{\mathbb{R}} \phi_{1}(\eta)\left(\int_{\eta}^{\infty} \phi_{2}(y) d y\right) d \eta$. After choosing $\theta_{i}$ and $\phi_{i}(i=1,2)$ such that $A=\frac{u_{1}}{u_{1}-u_{2}}$ and $B=\frac{v_{1}}{v_{1}-v_{2}}$, from (3.17) and (3.18) one gets at once that for $\psi \in C_{0}^{\infty}\left(\mathbb{R}^{2}\right)$

$$
\begin{aligned}
& \lim _{\varepsilon \rightarrow 0} \iint_{\mathbb{R}^{2}}\left[\bar{R}_{u}(\varepsilon, \xi, \eta)\left((\xi \psi)_{\xi}+(\eta \psi)_{\eta}\right)-\bar{R}_{u}(\varepsilon, \xi, \eta) \bar{R}_{v}(\varepsilon, \xi, \eta) \psi_{\eta}(\xi, \eta)\right] d \xi d \eta \\
& \quad=-\rho^{2} A B \psi_{\eta}(0,0) .
\end{aligned}
$$

Similarly,

$$
\begin{aligned}
& \lim _{\varepsilon \rightarrow 0} \iint_{\mathbb{R}^{2}}\left[\bar{R}_{v}(\varepsilon, \xi, \eta)\left((\xi \psi)_{\xi}+(\eta \psi)_{\eta}\right)-\bar{R}_{u}(\varepsilon, \xi, \eta) \bar{R}_{v}(\varepsilon, \xi, \eta) \psi_{\xi}(\xi, \eta)\right] d \xi d \eta \\
& \quad=-\rho^{2} A B \psi_{\xi}(0,0)
\end{aligned}
$$

Thus, $(\bar{U}, \bar{V}) \in \mathcal{G}\left(\mathbb{R}^{2}\right)$, with $\bar{R}_{u}$ and $\bar{R}_{v}$ as their respective elements, is not a solution to $(1.1),(1.2)$.

Indeed, at this time, not only is the solution to (1.1),(1.2) singular on the half lines $\xi=0, \eta>0$ and $\eta=0, \xi>0$, but also singular at the point $(0,0)$. The right-handed sides of (3.19) and (3.20) contain information enough to make us give the expressions of the solution to $(1.1),(1.2)$ at the point $(0,0)$. In fact, we define two distributions $\mu_{1}$ and $\mu_{2}$ supported on the origin

$$
\left\langle\mu_{1}, \psi\right\rangle=c_{1} \psi_{\eta}(0,0) \quad \text { and } \quad\left\langle\mu_{2}, \psi\right\rangle=c_{2} \psi_{\xi}(0,0)
$$

for $\psi \in C_{0}^{\infty}\left(\mathbb{R}^{2}\right)$, where $c_{1}$ and $c_{2}$ are constants to be determined below. We set $U, V \in \mathcal{G}\left(\mathbb{R}^{2}\right)$ with $R_{u}$ and $R_{v}$ as their representatives, respectively. Here $R_{u}$ and $R_{v}$ are determined by

$$
\begin{aligned}
R_{u}(\varepsilon, \xi, \eta) & =\bar{R}_{u}(\varepsilon, \xi, \eta)+\left(\mu_{1} * w_{3 \varepsilon}\right)(\xi, \eta) \\
& =\bar{R}_{u}(\varepsilon, \xi, \eta)+c_{1} \cdot \frac{1}{\varepsilon^{3}} \theta_{3}\left(-\frac{\xi}{\varepsilon}\right) \phi_{3}^{\prime}\left(-\frac{\eta}{\varepsilon}\right) \\
R_{v}(\varepsilon, \xi, \eta) & =\bar{R}_{v}(\varepsilon, \xi, \eta)+\left(\mu_{2} * w_{4 \varepsilon}\right)(\xi, \eta) \\
& =\bar{R}_{v}(\varepsilon, \xi, \eta)+c_{2} \cdot \frac{1}{\varepsilon^{3}} \theta_{4}^{\prime}\left(-\frac{\xi}{\varepsilon}\right) \phi_{4}\left(-\frac{\eta}{\varepsilon}\right)
\end{aligned}
$$

where $w_{i \varepsilon}(\xi, \eta)=\frac{1}{e^{3}} \theta_{i}\left(\frac{\xi}{\varepsilon}\right) \phi_{i}\left(\frac{\eta}{\varepsilon}\right)$ and $\theta_{i}, \phi_{i} \in C_{0}^{\infty}(\mathbb{R})$ with $\int_{\mathbb{R}} \theta_{i}(\xi) d \xi=\int_{\mathbb{R}} \phi_{i}(\xi) d \xi=$ $1(i=3,4)$.

It remains to verify that $R_{u}$ and $R_{v}$ given by (3.22) satisfy (3.1) and (3.2) for 
$\psi \in C_{0}^{\infty}\left(\mathbb{R}^{2}\right)$. Actually, from (3.22) we have that for $\psi \in C_{0}^{\infty}\left(\mathbb{R}^{2}\right)$

$$
\begin{aligned}
& \iint_{\mathbb{R}^{2}} R_{u}(\varepsilon, \xi, \eta) \psi(\xi, \eta) d \xi d \eta \\
& =\iint_{\mathbb{R}^{2}} \bar{R}_{u}(\varepsilon, \xi, \eta) \psi(\xi, \eta) d \xi d \eta+c_{1} \cdot \frac{1}{\varepsilon^{3}} \iint_{\mathbb{R}^{2}} \psi(\xi, \eta) \theta_{3}\left(-\frac{\xi}{\varepsilon}\right) \phi_{3}^{\prime}\left(-\frac{\eta}{\varepsilon}\right) d \xi d \eta \\
& =\iint_{\mathbb{R}^{2}} \bar{R}_{\mathbf{u}}(\varepsilon, \xi, \eta) \psi(\xi, \eta) d \xi d \eta+c_{1} \cdot \frac{1}{\varepsilon} \iint_{\mathbb{R}^{2}} \psi(-\varepsilon \xi,-\varepsilon \eta) \theta_{3}(\xi) \phi_{3}^{\prime}(\eta) d \xi d \eta \\
& =\iint_{\mathbb{R}^{2}} \bar{R}_{u}(\varepsilon, \xi, \eta) \psi(\xi, \eta) d \xi d \eta+c_{1} \psi_{\eta}(0,0)+O(\varepsilon)
\end{aligned}
$$

since $\psi(-\varepsilon \xi,-\varepsilon \eta)=\psi(0,0)-\varepsilon \xi \psi_{\xi}(0,0)-\varepsilon \eta \psi_{\eta}(0,0)+O\left(\varepsilon^{2}\right)$, while

$$
\begin{aligned}
\iint_{\mathbb{R}^{2}} & R_{u}(\varepsilon, \xi, \eta) R_{v}(\varepsilon, \xi, \eta) \psi(\xi, \eta) d \xi d \eta \\
= & \iint_{\mathbb{R}^{2}} \bar{R}_{u}(\varepsilon, \xi, \eta) \bar{R}_{v}(\varepsilon, \xi, \eta) \psi(\xi, \eta) d \xi d \eta \\
& +c_{1} \cdot \frac{1}{\varepsilon^{3}} \iint_{\mathbb{R}^{2}} \psi(\xi, \eta) \theta_{3}\left(-\frac{\xi}{\varepsilon}\right) \phi_{3}^{\prime}\left(-\frac{\eta}{\varepsilon}\right) \bar{R}_{v}(\varepsilon, \xi, \eta) d \xi d \eta \\
& +c_{2} \cdot \frac{1}{\varepsilon^{3}} \iint_{\mathbb{R}^{2}} \psi(\xi, \eta) \theta_{4}^{\prime}\left(-\frac{\xi}{\varepsilon}\right) \phi_{4}\left(-\frac{\eta}{\varepsilon}\right) \bar{R}_{u}(\varepsilon, \xi, \eta) d \xi d \eta \\
& +c_{1} c_{2} \frac{1}{\varepsilon^{6}} \iint_{\mathbb{R}^{2}} \psi(\xi, \eta) \theta_{3}\left(-\frac{\xi}{\varepsilon}\right) \theta_{4}^{\prime}\left(-\frac{\xi}{\varepsilon}\right) \phi_{3}^{\prime}\left(-\frac{\eta}{\varepsilon}\right) \phi_{4}\left(-\frac{\eta}{\varepsilon}\right) d \xi d \eta \\
& \sim \iint_{\mathbb{R}^{2}} \bar{R}_{u}(\varepsilon, \xi, \eta) \bar{R}_{v}(\varepsilon, \xi, \eta) \psi(\xi, \eta) d \xi d \eta+I_{1}^{\varepsilon}+I_{2}^{\varepsilon}+I_{3}^{\varepsilon} .
\end{aligned}
$$

Now we choose $\theta_{i}$ and $\phi_{i}(i=2,3)$ such that

$$
\left.\begin{array}{c}
\int_{\mathbb{R}} \theta_{2}(\xi) \theta_{3}(\xi) d \xi=\int_{\mathbb{R}} \xi \theta_{2}(\xi) \theta_{3}(\xi) d \xi=0 \\
\int_{\mathbb{R}}^{\infty} \phi_{2}(\xi) \phi_{3}(\xi) d \xi=0 \\
\int_{\mathbb{R}} \theta_{3}(\xi)\left(\int_{\xi}^{\infty} \theta_{2}(x) d x\right) d \xi \cdot \int_{\mathbb{R}} \eta \phi_{3}^{\prime}(\eta)\left(\int_{\eta}^{\infty} \phi_{2}(y) d y\right) d \eta=\frac{v_{1}}{v_{2}-v_{1}} .
\end{array}\right\}
$$

This can be done, e.g., by taking $\theta_{2}, \theta_{3} \in C_{0}^{\infty}\left(\mathbb{R}^{2}\right)$ with supp $\theta_{2} \subset[0,1]$ and $\operatorname{supp} \theta_{3} \subset$ $[-1,0]$, and $\rho_{i} \in C_{0}^{\infty}\left(\mathbb{R}^{2}\right)$ with $\int_{\mathbb{R}} \rho_{i}(\eta) d \eta=1 \quad(i=1,2)$, supp $\rho_{1} \subset[-2,-1]$ and supp $\rho_{2} \subset[1,2]$. Set $\phi_{3}=\theta_{2}$ and $\phi_{2}=(1-B) \rho_{1}+B \rho_{2}$, with $B=\frac{v_{1}}{v_{1}-v_{2}}$. From (3.16) and (3.25) it follows that

$$
I_{1}^{\varepsilon}=c_{1} \cdot \frac{1}{\varepsilon^{3}} \iint_{\mathbb{R}^{2}} \psi(\xi, \eta) \theta_{3}\left(-\frac{\xi}{\varepsilon}\right) \phi_{3}^{\prime}\left(-\frac{\eta}{\varepsilon}\right) \bar{R}_{v}(\varepsilon, \xi, \eta) d \xi d \eta
$$


754 Jiaxin $\mathrm{Hu}$

$$
\begin{aligned}
= & \frac{c_{1}}{\varepsilon} \iint_{\mathbb{R}^{2}} \psi(-\varepsilon \xi,-\varepsilon \eta) \theta_{3}(\xi) \phi_{3}^{\prime}(\eta) \bar{R}_{v}(\varepsilon,-\varepsilon \xi,-\varepsilon \eta) d \xi d \eta \\
= & \frac{c_{1}}{\varepsilon} \iint_{\mathbb{R}^{2}} \psi(-\varepsilon \xi,-\varepsilon \eta) \theta_{3}(\xi) \phi_{3}^{\prime}(\eta)\left[v_{1}+\left(v_{2}-v_{1}\right) \int_{\xi}^{\infty} \theta_{2}(x) d x \int_{\eta}^{\infty} \phi_{2}(y) d y\right. \\
& \left.+\rho b \theta_{2}(\xi) \int_{\eta}^{\infty}(y-\eta) \phi_{2}(y) d y-\frac{\rho}{\varepsilon} \theta_{2}(\xi) \int_{\eta}^{\infty} \phi_{2}(y) d y\right] d \xi d \eta \\
= & \frac{c_{1}}{\varepsilon} \iint_{\mathbb{R}^{2}}\left(\psi(0,0)-\varepsilon \xi \psi_{\xi}(0,0)-\varepsilon \eta \psi_{\eta}(0,0)+O\left(\varepsilon^{2}\right)\right) \theta_{3}(\xi) \phi_{3}^{\prime}(\eta) \\
& \times\left\{v_{1}+\left(v_{2}-v_{1}\right) \int_{\xi}^{\infty} \theta_{2}(x) d x \int_{\eta}^{\infty} \phi_{2}(y) d y\right. \\
& \left.+b \rho \theta_{2}(\xi) \int_{\eta}^{\infty}(y-\eta) \phi_{2}(y) d y\right\} d \xi d \eta \\
& -\frac{\rho c_{1}}{\varepsilon^{2}} \iint_{\mathbb{R}^{2}}^{\theta_{2}(\xi) \theta_{3}(\xi) \phi_{3}^{\prime}(\eta)} \int_{\eta}^{\infty} \phi_{2}(y) d y \cdot\left\{\psi(0,0)-\varepsilon \xi \psi_{\xi}(0,0)\right. \\
& -\varepsilon \eta \psi_{\eta}(0,0)+\frac{1}{2} \varepsilon^{2} \xi^{2} \psi_{\xi \xi}(0,0)+\varepsilon^{2} \xi \eta \psi_{\xi \eta}(0,0) \\
& \left.+\frac{1}{2} \varepsilon^{2} \eta^{2} \psi_{\eta \eta}(0,0)+O\left(\varepsilon^{3}\right)\right\} d \xi d \eta \\
= & O(\varepsilon) .
\end{aligned}
$$

Similarly, we have that

$$
I_{2}^{e}=c_{2} \cdot \frac{1}{\varepsilon^{3}} \iint_{\mathbb{R}^{2}} \psi(\xi, \eta) \theta_{4}^{\prime}\left(-\frac{\xi}{\varepsilon}\right) \phi_{4}\left(-\frac{\eta}{\varepsilon}\right) \bar{R}_{u}(\varepsilon, \xi, \eta) d \xi d \eta=O(\varepsilon)
$$

if we set

$$
\left.\begin{array}{c}
\int_{\mathbb{R}} \phi_{1}(\eta) \phi_{4}(\eta) d \eta=\int_{\mathbb{R}} \eta \phi_{1}(\eta) \phi_{4}(\eta) d \eta=0 \\
\int_{\mathbb{R}} \theta_{1}(\xi) \theta_{4}(\xi) d \xi=0 \\
\int_{\mathbb{R}} \phi_{4}(\eta)\left(\int_{\eta}^{\infty} \phi_{1}(y) d y\right) d \eta \cdot \int_{\mathbb{R}} \xi \theta_{4}^{\prime}(\xi)\left(\int_{\xi}^{\infty} \theta_{1}(x) d x\right) d \xi=\frac{u_{1}}{u_{2}-u_{1}}
\end{array}\right\}
$$

and

$$
\begin{aligned}
I_{3}^{\varepsilon} & =c_{1} c_{2} \frac{1}{\varepsilon^{6}} \iint_{\mathbb{R}^{2}} \psi(\xi, \eta) \theta_{3}\left(-\frac{\xi}{\varepsilon}\right) \theta_{4}^{\prime}\left(-\frac{\xi}{\varepsilon}\right) \phi_{3}^{\prime}\left(-\frac{\eta}{\varepsilon}\right) \phi_{4}\left(-\frac{\eta}{\varepsilon}\right) d \xi d \eta \\
& =O(\varepsilon)
\end{aligned}
$$


if we set

$$
\int_{\mathbb{R}} \xi^{k} \theta_{3}(\xi) \theta_{4}^{\prime}(\xi) d \xi=0 \quad \text { or } \quad \int_{\mathbb{R}} \eta^{k} \phi_{3}^{\prime}(\eta) \phi_{4}(\eta) d \eta=0 \quad(k=0,1,2,3,4) .
$$

Combining (3.26) - (3.28), we deduce from (3.24) that

$$
\begin{aligned}
& \iint_{\mathbb{R}^{2}} R_{u}(\varepsilon, \xi, \eta) R_{v}(\varepsilon, \xi, \eta) \psi(\xi, \eta) d \xi d \eta \\
& \quad=\iint_{\mathbb{R}^{2}} \bar{R}_{u}(\varepsilon, \xi, \eta) \bar{R}_{v}(\varepsilon, \xi, \eta) \psi(\xi, \eta) d \xi d \eta+O(\varepsilon) .
\end{aligned}
$$

Therefore, (3.23), (3.29) and (3.19) yield that

$$
\begin{aligned}
\lim _{\varepsilon \rightarrow 0} \iint_{\mathbb{R}^{2}}\left[R_{u}(\varepsilon, \xi, \eta)\left((\xi \psi)_{\xi}+(\eta \psi)_{\eta}\right)-R_{u}(\varepsilon, \xi, \eta) R_{v}(\varepsilon, \xi, \eta) \psi_{\eta}\right] d \xi d \eta \\
=\lim _{\varepsilon \rightarrow 0} \iint_{\mathbb{R}^{2}}\left[\bar{R}_{u}(\varepsilon, \xi, \eta)\left((\xi \psi)_{\xi}+(\eta \psi)_{\eta}\right)\right. \\
\left.\quad-\bar{R}_{u}(\varepsilon, \xi, \eta) \bar{R}_{v}(\varepsilon, \xi, \eta) \psi_{\eta}(\xi, \eta)\right] d \xi d \eta+3 c_{1} \psi_{\eta}(0,0) \\
=\left(3 c_{1}-\rho^{2} A B\right) \psi_{\eta}(0,0) \\
=0
\end{aligned}
$$

for $\psi \in C_{0}^{\infty}\left(\mathbb{R}^{2}\right)$ if $c_{1}=\frac{1}{3} \rho^{2} A B$. In the same way, we have that $\psi \in C_{0}^{\infty}\left(\mathbb{R}^{2}\right)$

$$
\lim _{\varepsilon \rightarrow 0} \iint_{\mathbb{R}^{2}}\left[R v(\varepsilon, \xi, \eta)\left((\xi \psi)_{\xi}+(\eta \psi)_{\eta}\right)-R_{u}(\varepsilon, \xi, \eta) R_{v}(\varepsilon, \xi, \eta) \psi_{\xi}\right] d \xi d \eta=0
$$

for the same $\theta_{i}$ and $\phi_{i} \quad(i=2,3,4)$ as above if $c_{2}=\frac{1}{3} \rho^{2} A B$. This verifies that $U, V \in \mathcal{G}\left(\mathbb{R}^{2}\right)$, with $R_{u}$ and $R_{v}$ as their representatives, respectively, satisfy (1.1) in the sense of association. The initial condition (2.4) is easily seen. We omit the details.

Remarks. 3. The approximation process above guarantees that $T_{i} \mu_{j}=0 \quad(i, j=$ 1,2 ), but $T_{1} T_{2} \neq 0$ (when $u_{1} v_{1} \neq 0$ ). One can choose other approximations to define the product of $\bar{U}$ and $\bar{V}$ so that not only $T_{i} \mu_{j}=0 \quad(i, j=1,2)$ but also $T_{1} T_{2}=0$. However, it is more reasonable to define $T_{1} T_{2} \neq 0$ when $u_{1} v_{1} \neq 0$ since the intersection of the supports of $T_{1}$ and $T_{2}$ is non-void. The behavior of the solution to (1.1),(1.2) at the origin should be considered.

4. It is easily seen that $U, V \in \mathcal{G}\left(\mathbb{R}^{2}\right)$ have $\bar{u}(\xi, \eta)+\rho T_{1}+c_{1} \mu_{1}$ and $\bar{v}(\xi, \eta)+\rho T_{2}+c_{2} \mu_{2}$ as their respective macroscopic aspects.

We conclude this paper with the following

Theorem. The non-classical Riemann solutions constructed in [6-8] satisfy (1.1) in the sense of associaton.

Acknowledgement. I am deeply grateful to Prof. Xiaqi Ding for his persistent encouragement. Also I would like to express my thanks to Prof. M. Oberguggenberger for bringing his works to my attention. Finally, my heart-felt thanks are due to the anonymous referee for his keen observations and helpful suggestions, which resulted in (2.4). 


\section{References}

[1] Colombeau, J. F.: New Generalized Functions and Multiplication of Distributions. Amsterdam: North Holland 1984.

[2] Colombeau, J. F.: Multiplication of Distributions. Lect. Notes Math. 1532 (1992), 1 184.

[3] Colombeau, J. F. and A. Y. LeRoux: Multiplication of distributions in elasticity and hydrodynamics. J. Math. Phys. 29 (1988), $315-319$.

[4] Colombeau, J. F., LeRoux, A. Y., Noussair, A. and B. Perrot: Microscopic profiles of shock waves and ambiguities in multiplications of distributions. SIAM J. Numer. Anal. 26 (1989), $871-883$.

[5] Egorov, Yu. V.: On the theory of generalized functions. Russ. Math. Surveys 45 (1990), $3-40$.

[6] Hu, J. X.: The Riemann Problem for a Two-Dimensional Hyperbolic System of Nonlinear Conservation Laws with functional solutions. Acta Math. Sci. 18 (1998), 45 - 56.

[7] Hu, J. X.: The Riemann problem for a two-dimensional hyperbolic system of conservation laws with non-classical shock waves: some one-J and non- $R$ initial data. Acta Math. Sci. 4 (1997), $361-375$.

[8] $\mathrm{Hu}, \mathrm{J}$. X.: The Riemann problem for a resonant nonlinear system of conservation laws containing Dirac-measure solutions. Proc. Royal Soc. Edin. 128 (1998), 81 - 94.

[9] Leveque, R. J., Peskin, C. S. and P. D.Lax: Solution of a two-dimensional cochlea model using transform techniques. SIAM J. Appli. Math. 45 (1985), $450-464$.

[10] Oberguggenberger, M.: Multiplication of Distributions and Applications to Partial Differential Equations (Pitman Res. Notes Math.: Vol. 259). Harlow: Longman 1992.

[11] Oberguggenberger, M.: Products of distributions: nonstandard methods. Z. Anal. Anw. 7 (1988), 347 - 365; Corrections to this article in: Z. Anal. Anw. 10 (1991), $263-264$.

[12] Rosinger, E. E.: Nonlinear Partial Differential Equations. An Algebraic. View of Generalized Solutions. Amsterdam: North Holland 1990.

[13] Schwartz, L.: Sur l'impossibilite de la multiplication des distributions. C.R. Acad. Sci. Paris 239 (1954), $847-848$.

[14] Smoller, J.: Shock Waves and Reaction-Diffusion Equations. New York: Springer-Verlag 1983.

[15] Todorov, T.: Colombeau's generalized functions and nonstandard analysis. In: Generalized Functions, Convergence Structures and Their Applications(eds.: B. Stankovic et al.). New York: Plenum Press 1988, pp. 327 - 339.

Added in proof:

[16] Hayes, B. T and P. G. LeFloch: Measure solutions to a strictly system of conservation laws. Nonlinearity 9 (1996), $1547-1563$.

[17] Hu, J. X.: A limiting viscosity approach to Riemann solutions containing Delta-shock waves for nonstrictly hyperbolic conservation laws. Quart. Appl. Math. 3 (1996), 361 373.

[18] Keyfitz, B. L. and H. C. Kranzer: Spaces of weighted measures for conservation laws with singular shock solutions. J. Diff. Equ. 118 (1995), 420 - 451.

[19] Maso, G. D., LeFloch, P. G. and F. Murat: Definition and weak stability of nonconservative products. J. Math. Pure Appl. 74 (1995), 483 - 548. 
[20] Tan, D. C. and T. Zhang: Two-dimensional Riemann problem for a hyperbolic system of nonlinear conservation laws. J. Diff. Equ. 111 (1994), $203-254$ and $255-282$.

[21] Tan, D. C., Zhang, T. and Y. X. Zheng: Delta-shock waves as limits of vanishing viscosity for hyperbolic systems of conservation laws. J. Diff. Equ. 112 (1994), 1 - 32.

Received 02.07.1997; in revised form 08.04.1998 